\title{
FOURTH POSTER DISCUSSION
}

KING: For convenience I have tried to group the papers of this section into a logical order by subject matter. The first group deals with surface photometry, in the papers A Multicolor CCD Survey of Southern Globular Clusters and The Structure of Collapsed Cluster Cores. I think that we should also include in our discussion the related material from George Djorgovski's invited paper this morning. All three of these studies are quite similar in their methods, and they even have a number of clusters in common. As far as I can see, their observations agree with each other rather well; where there are differences, they are differences of interpretation. Forte and Mendez put more emphasis on small deviations than I would, but I suspect that Djorgovski might agree more with them.

COHEN: Does your new set of $U$ frames indicate the presence of cusps in those clusters where Djorgovski \& King saw central cusps?

GRINDLAY: To the extent we have analyzed our new UBV CCD data beyond the first clusters reported in our paper, they agree with the Djorgovski and King results. We have good data on all of their candidate cusp clusters and are now deriving U-profiles and model fits for each.

LARSON: Djorgovski said this morning that globular clusters come in two "flavors", with and without cusps. Could there actually be a continuum of properties in this respect?

DJORGOVSKI: Yes, it is clearly possible that there are weak or invisible cusps in King-model-like clusters. That may be the explanation for high-concentration clusters, like NGC 5824, which should have collapsed by now, but look like "perfect" King models. In most cases one can clearly classify the profiles as either King model or PCC, but there are some intermediate or noisy cases, where we cannot tell.

RING: George and I work together, but we don't necessarily agree about everything. I do think that we are likely to have real intermediate cases. A problem, though, is that some of our data are rather noisy.

GRINDLAY: I agree (with Ivan) that cusps could be missed (even in U) in some clusters. The available resolution (both seeing and detector) is obviously important, and further cusps may become evident with studies such as the one we have initiated at the CFHT with both better seeing and the double density CCD chip. The factor-of-two pixel resolution is important.

KING: The next set of papers all deal with velocities, although they are rather different from each other. The paper Radial Velocity Study 
of NGC 6712 deals with radial velocities in one cluster and notes that the low velocity dispersion implies that there are not a lot of massive remnants, whereas the cluster has an X-ray source. A Survey of Globular Cluster Velocity Dispersions fits isotropic-velocity models to a number of clusters and derives values of $\mathrm{M} / \mathrm{L}$, which do not appear to correlate with anything. This does not directly contradict the much-discussed result of McClure et al., but it does lead me to wonder a little. Anisotropy in Omega Centauri and 47 Tucanae fits detailed models to two clusters and determines values of the anisotropy. Here I was pleased to see that Omega Centauri, which has about 30 times as long a relaxation time, has much more anisotropy than 47 Tucanae.

WEBBINK: Regarding the lack of correlation of $\mathrm{M} / \mathrm{L}$ with anything else, as discussed in the poster paper by Pryor et al., this same effect appears in Illingworth's survey. The cluster-to-cluster variations appear to be real. I realize you do not have a very large sample of clusters, but did you look for a correlation with a combination of parameters?

PRYOR: I have checked the physically meaningful combinations that I could think of and found no correlations. If you have any suggestions of quantities to check, I would like to hear them.

KING: Did you include other people's velocities in your paper?

PRYOR: The clusters on our poster are only those for which we have obtained data. However, including Illingworth's clusters does not change the conclusion that there is no apparent correlation between $\mathrm{M} / \mathrm{L}$ and metallicity.

PENNY: The velocity anisotropies deduced by Meylan for $\omega$ Cen are of the form that would reduce the discrepancies between the radial velocities and proper motions in M 22 found by Kyle Cudworth.

CUDWORTH: 1) The M 22 proper motions are within a few core radii of the cluster center, where anisotropy is not expected. We looked for and did not find evidence for anisotropy in the proper motion data. 2) Proper motions in 47 Tuc are currently being reduced and will go out to a larger radius than the radial velocity data that has been published.

KING: Now that we have observed values of the anisotropy in $\omega$ Cen, 47 Tuc, $M 3$, and $M 13$, it ought to be possible to compare them with the relaxation times and predict the anisotropies in other clusters.

KING: The next set of papers deals with dynamical evolution of clusters. Evolution of Globular Clusters Including a Degenerate Component and Evolution of Globular Clusters with Tidally-Captured Binaries through Core Collapse deal with post-core-collapse evolution, including the effect of binaries; The Formation and Evolution of Three-Body Binaries in Evolved Globular Clusters and Tidal Effects on Stellar Evolution in Close Binaries Formed in Globular Clusters treat 
the formation and behavior of the binaries themselves; and The Effects of Stellar Evolution on Globular Cluster Dynamical Evolution follows evolution with a number of effects operating simultaneously.

KING: Would one of the authors please contrast the approaches of the first two papers?.

LEE, H. M.: The paper by Statler et al. showed that, using a single component model, tidally captured binaries can reverse the core collapse well before any dynamically important three-body binaries form. In my paper, the massive degenerate stars are included to get a wide range of solutions for post-collapse clusters. Especially, some clusters with very small cores (e.g., M 15) can be accounted for by this model.

KING: The paper by McMillan seems to increase the effect of binaries. Is that right?

MCMILLAN: The main point I wanted to make in this paper is that, if you want to include "three-body" binaries correctly in a simulation, it is inadequate only to consider three-body encounters. I find that as many as fifty percent of the encounters involving binaries (formation and subsequent interactions) involve a total of four or more stars and this has a substantial effect on the hardening and heating rates, even for quite hard pairs.

KING: The paper by Ray and Kembhavi improves the calculations of the tidal formation of binaries. In this connection, Bob Mathieu has an interesting empirical result on the rate at which orbits circularize.

MATHIEU: Two years ago Mayor and Mermilliod noted that all $\sim 1 \mathrm{M}_{0}$ binaries in the Hyades with periods of less than $5.7^{\mathrm{d}}$ had circular orbits, while those with longer periods all had eccentric orbits. They attributed this cutoff to tidal circularization processes - at 0.8 billion years binaries with periods less than 5.7 days have had time to tidally circularize. We have recently completed our radial velocity survey of M 67 and find a similar cutoff, but at 10.7 days. This longer period cutoff is in the expected sense given at tidal circularization integration. In fact, this result argues for a new clock with which to date clusters, one that is independent of stellar evolution theory and, when used in a relative sense, is also not sensitive to stellar interiors theory. Tsevi Mazeh and $I$ have developed the clock mechanism from the circularization theory of Lecar et al. and applied it to the Hyades and M 67. Our preliminary results are ages of $0.2-0.4$ billion years for the Hyades and $5-7.5$ billion years for $M 67$, to be compared with stellar evolution ages of 0.8 billion and 5 billion years respectively. Ivan was not so very impressed with the accuracy, but I find it quite encouraging given the primitive state of the clock mechanism! More to the point at this session, the agreement is remarkable given the very simple state of 
tidal circularization theory; Lecar et al. expected at best order of magnitude accuracy from their theory. Our observations indicate that the theory may be in better shape than we had a right to expect, and add support to the application of similar theory to the problem of tidal-capture binaries. We are extending our binary survey to the young cluster M 35, where we predict a period cutoff of about 3 days.

KING: Would Dave Chernoff please explain the relation of his paper to his other papers at this symposium?

CHERNOFF: This paper explains the precollapse evolution of a globular cluster under the influence of 1) a tidal boundary 2) a mass spectrum and 3) stellar evolution. The results indicate that for a moderately bound cluster ( $\mathrm{W}_{0}=3$ ) of $10^{5} \mathrm{M}_{0}$ at the galactocentric location a substantial fraction of mass is lost from the cluster across the tidal boundary and collapse is significantly delayed.

CAPUZZO-DOLCETTA: With regard to Chernoff's paper on the influence of stellar evolution on globular cluster dynamics, I would point out that a lot of work (and results) on this topic were obtained several years ago by Angeletti, Giannone and me and published in the journals Astron. Astrophys. and Astrophys and Space Sci.

KING: The next set of papers deals with distributions that are segregated, presumably by stellar mass. The Spatial Distribution of Spectroscopic Binaries looks at binaries and blue stragglers in the "honorary globular cluster" M 67; Evidence for Mass Segregation in NGC 5466 looks at blue stragglers in NGC 5466 (and adds some preliminary information on NGC 5053); Origin and Radial Distribution of Faint Blue Horizontal-Branch Stars deals with faint BHB stars in M 15. There is a lot of agreement between these papers, about most of these stellar types being more centrally concentrated. The only thing we have seen that doesn't seem to fit is the blue-straggler result mentioned previously by Flavio Fusi Pecci. I would like to give him a few minutes to try to reconcile the discrepancy.

FUSI PECCI: From a detailed comparison of the HB morphologies of the galactic globular clusters, M 15, NGC 5466 and M 92 we have shown (Buonanno et al. 1985, Astron. Astrophys. 145, 97) that the BHB stars found in the blue horizontal branch of M 15 fainter than the HB gap are "bona fide" HB stars (by considering the population ratios over complete samples of the different branches and the different clusters). We have also proposed as a working hypothesis a naive interpretation of both the peculiar spatial distribution of blue HB stars we find in M 15 and in NGC 6752 (BHB stars are more frequent in the outer regions). The basic idea is that the central stellar density of the cluster (coupled with core rotation and the mass loss of each individual star) may have induced on each star subtle differential effects visible only during "special" evolutionary phases, such as the HB phase is. According to this suggestion one would expect (as a zeroth approximation): $i$, high stellar density: BHB stars more frequent in 
the outer regions; ii, low stellar density: BHB stars uniformly distributed or more frequent in central regions of the cluster.

GRINDLAY: I think the main point of disagreement between us is whether the radial dependence of faint blue horizontal-branch stars can even be discussed for very dense clusters, such as M 15 or NGC 6752. FBHB stars can simply not be recognized in the central few core radii of M 15 (although we are trying to do so with new high resolution CFHT observations) whereas they can be studied in lower density clusters such as, $\omega$ Cen. Dynamical mass segregation effects are expected to only be significant for the central few core radii.

NEMEC: A recent CM diagram for NGC 4833 (Nemec, Richer \& Fahlman, 1986, in prep.), a relatively open, metal-poor globular cluster, shows a very faint blue horizontal branch. Does this, then, represent an exception to your trend that extended blue horizontal branches go with low concentration class?

FUSI PECCI: Is this connected with the existence and spatial distribution of blue stragglers? It is hard to answer as: $i$, many aspects may play a role: binary existence, frequency and origin; cluster central density; mass loss; metallicity; etc.; $i$, the observational data are still insufficient to present any reliable scenario: NGC 5466, NGC 5053 - low stellar density - have BS with a spatial distribution peaked at the cluster center (Nemec et al., this Symposium), M 3 - high stellar density - displays a high BS frequency in the outer regions (Buonanno et al., this symposium). My present opinion is that: $i$, the interpretation of $\mathrm{HB}$ morphology and HB star spatial distribution does not require mass segregation or the existence of a signigicant population of binaries, $i$, BS presence and spatial distribution do probably require or imply both of them.

HESSER: To Grindlay: Have you examined the images of your blue stars in $\omega$ Cen, which were presumably located by an automatic star finding routine? Did their images look abnormal?

GRINDLAY: Yes, we have looked at the field blue horizontal-branch star images and they are real. We have also obtained spectra at CTIO on several of these stars and verified that they are indeed cluster FBHB stars.

KING: The next set of papers deals with particular kinds of stars, and to some extent with their origins. In discussing them, we should keep the preceding set in mind too. Variability of Omega Centauri Blue Stragglers: Clues to their Origin is an interesting attempt to connect blue stragglers with dwarf Cepheids, which are another kind of mystery star; and A Search for Optical Counterparts of Globular Cluster X-ray Sources is actually a pair of papers, one dealing with the identified optical counterpart of the X-ray source in $M 15$ and the other reporting on a less successful examination of some other clusters. And I think that along with these papers we should again take note of the paper 
Candidate for the Recovered Nova of 1938 in the Globular Cluster $M 14$ on the M 14 nova.

WEBBINK: The position of the old nova candidate in $M 14$ in the HR diagram of that cluster would imply $M_{y} \sim+3$. Every other old nova this bright is extremely blue, making the color of this star (roughly that of a late $\mathrm{K}$ dwarf) quite anomalous.

MATHIEU: I have a question for Ron Webbink that I tried to ask him in Delhi, but I couldn't find him! Ron, what do you think blue stragglers are?

WEBBINK: I have no strong convictions regarding their true nature, though I don't think any of the alternatives are more compelling than the binary hypothesis. I would point out that the occurrence of blue stragglers more than two magnitudes above turnoff is not necessarily evidence against a binary origin, although it is frequently cited as such. A star which increases rapidly in mass - on a thermal timescale or faster - becomes very oversized and overluminous for its mass. Thus, if mass transfer or coalescence occurs fast enough, blue stragglers well above the two magnitude limit can be created, but the ratio of the number of blue stragglers more than two magnitudes above turnoff to the total number of blue stragglers should not exceed the ratio of thermal to nuclear timescales for those stars.

KING: Isn't that ratio quite small?

WEBBINK: It's about $1 / 10$.

MCMILIAN: Another possible way of producing an anomalously blue star is the tidal capture process itself. After capture and circularization, the pulsational energy deposited in the component stars is radiated away. I have found that excess luminosities of over a hundred times that of the unheated star are quite possible. The star's response to this extra heat, which is deposited primarily near the surface, is a substantial increase (factor of two or three) in surface temperature, although an increase in radius ( 50z-100z) also occurs. Thus, a much brighter, bluer object results.

KING: The next set of papers deals with the properties of X-ray sources in globular clusters, and also of the possibility of a related hot gas. Low Luminosity Globular Cluster X-ray Sources looks to me like a rather definitive discussion of low-luminosity X-ray sources in globular clusters. Exosat Observations of Omega Centauri reports four separate kinds of $\mathrm{X}$-ray information for Omega Centauri, and New Methods of Search for a Hot Gas in Clusters discusses how hot gas might be looked for. I would like to start the discussion by asking Paul Hertz whether there is any correlation between low-luminosity $\mathrm{X}$-ray sources and the presence of a central cusp. 
HERTZ: A quick comparison shows no correlation. Low luminosity X-ray sources are correlated with tidal capture timescales. The presence of cusps may also be so correlated, but not at a level significant enough to convince anyone who knows anything about statistics.

KRON: M 22 is in the IRAS faint source catalog and is bright at $12.5 \mu$.

COHEN: There is a previously undiscovered planetary nebula near the core of M 22 which is the IRAS point source (Neugebauer, Soifer, Gillett \& Cohen, to be published).

KING: Next we have some more theoretical papers, which I separated out because they seem to be of a more abstract nature. Radial Velocity Profiles for Anisotropic Symmetric Clusters: An Example is an impressive but complicated discussion of a family of Plummer models. Personally I have always been peeved by Plummer models, because they don't look like real clusters at all. My first reaction to this paper was like that of the French general who witnessed the charge of the Light Brigade: "C'est magnifique, mais ce n'est pas la guerre!" But then I looked at it in a more responsible way. I think that the indeterminacies pointed out by de Jonghe must apply in a similar way to more realistic cluster models, and we should take serious note of the problem. Linear Density Waves in Globular Clusters deals with the possibility of density waves in globular clusters, on which I'm not capable of commenting. On Gravothermal Oscillations is far too long and difficult for me to have understood it in the brief time available, so I would like to ask Jeremy Goodman if he will make some brief remarks about it.

GOODMAN: I would like to emphasize that postcollapse clusters are expected to undergo large-amplitude oscillations in central density on a timescale of about one half-mass relaxation time. We do not yet know accurately how low the central density may become during the re-expansion phase (better calculations are needed), but it is possible that some apparently normal cores actually belong to postcollapse clusters. What was your question about M 67 here?

MATHIEU: M 67 is well fit by multi-mass King models, with no obvious irregularities in the core. But you must remember that the number of stars is small. If you look at Terlevich's or Aarseth's N-body simulations, while they show "oscillations" in the core, typically they involve only one or two binaries plus perhaps a few single stars. This would be difficult to distinguish. Indeed, several of our detected binaries are in the very center of the cluster and might be involved in such multiple-body interactions at the moment. (Jeremy then asked about the number of stars). The total mass of $M 67$ is of order 2000 $M_{0}$. The number of stars actually used in my dynamical analyses (set by the magnitude limit of $\mathrm{V}=19$ ) was about 800 .

KING: Regarding the paper by Goodman, it appears that a cluster that 
has core oscillations will spend most of its time in a puffed-up state. Should we observe something unusual then, or should the cluster look like an ordinary equilibrium state?

GOODMAN: These oscillating clusters are certainly in dynamical equilibrium (they obey Jeans' theorem), but not in thermodynamic equilibrium; but neither are King models. They do have approximately Maxwellian velocity distributions.

KING: Finally, we have three contributions related to problems of formation of globular clusters. Fragmentation of Proto-Globular Clusters discusses molecular processes that the authors believe are relevant, and The Formation of Globular Clusters and the First stellar Generation deals with successive stages of fragmentation. Formation of Population III Objects Due to Cosmic Strings tries to relate cosmic strings to cluster formation, and I can't make any intelligent comments on it at all. Do the authors of any of these papers have anything to add?

DI FAZIO: Just a short comment about my paper. It is interesting to note that the introduction of $\mathrm{H}_{2}$ formation and $\mathrm{H}_{2}$ cooling functions in the radiative calculations for the evolution of a proto-globular cluster and its fragmentation, does not alter dramatically the mass range of the mass spectrum of the first stellar generation formed. In fact, an isothermal phase is indeed reached at lower temperatures (due to $\mathrm{H}_{2}$ cooling processes), but the ambient gas exhaustion due to the fragmentation process itself raises the Jeans mass back up, halting the fragmentation. All this results in a range [40/1000] $\mathrm{M}_{0}$, instead of [80/3000] $M_{0}$ that was obtained (without $\mathrm{H}_{2}$ cooling) in a previous calculation for the fragmentation of proto-globular clusters (DiFazio, 1986, Astron. Astrophys. 159, 47).

ZINNECKER: I would just like to emphasize that there is a large uncertainty regarding the predicted physical conditions in the protogalaxy: Fall and Rees predict rather warm clouds, while we (Palla and Zinnecker) predict much cooler clouds due to strong molecular cooling. This is highlighted in our poster paper.

DJORGOVSKI: It is very interesting that the collapse time depends on the slope of the mass function. The slope according to McClure et al., correlates well with the metallicity. Ivan King and I checked whether there is any systematic difference in metallicity between the King model clusters and those with post-collapse cores. There isn't any. 